\title{
Uso de probiótico sobre a ativação de células T e controle de Salmonella Minnesota em frangos de corte ${ }^{1}$
}

INDEX TERMS: Probiotics, Enterococcus, immunity, lymphocytes, Lactobacillus, Salmonella Minnesota.

RESUMO.- Para avaliar o efeito do probiótico sobre a resposta imunológica de frangos de corte desafiados com Salmonella Minnesota (SM), 60 frangos foram divididos em três grupos: CN- (controle negativo) aves que não foram inoculadas com SM, CP- (controle positivo) aves inoculadas com SM e Probiótico- aves suplementadas na ração com probiótico composto de Lactobacillus acidophilus, L. plantarium, L. rhamnosus, L. bulgaricus, Enterococcus faecium, Streptococcus thermophilus e Bifidobacterium bifidum e desafiadas com SM. Aos 14 dias foi realizada a inoculação com SM e aos 7 e 35 dias foram quantificadas células caliciformes, CD4+ e CD8+ na mucosa intestinal do íleo e ceco. Aves suplementadas com probióticos aos 7 dias de idade apresentaram aumento significativo $(P \leq 0,05)$ de células caliciformes e CD4+ no íleo e

\footnotetext{
${ }^{1}$ Recebido em 7 de julho de 2012.

Aceito para publicação em 1 de outubro de 2012.

${ }^{2}$ Laboratório de Microbiologia e Ornitopatologia, Departamento de Medicina Veterinária, Universidade Federal do Paraná, Rua dos Funcionários 1540, Bairro Juvevê, Curitiba, PR 80035-050, Brasil. *Autor para correspondência: santin@ufpr.br
}

de células CD8+ no ceco. Aos 35 dias houve aumento significativo $(\mathrm{P} \leq 0,05)$ das células $\mathrm{CD} 8+$ nas aves inoculadas do $\mathrm{CN}$ e Probiótico. A utilização de probióticos proporcionou redução significativa $(\mathrm{P} \leq 0,05)$ da contagem de Salmonella sp.

TERMOS DE INDEXAÇÃO: Probióticos, Enterococcus, imunidade, linfócitos, Lactobacillus, Salmonella Minnesota.

\section{INTRODUÇÃO}

O intenso controle da ocorrência e disseminação de microrganismos patogênicos como bactérias do gênero Salmonella é fundamental para garantir a segurança dos produtos de origem avícola. No que se refere a Salmonella, os sorovares Enteritidis e Typhimurium são os mais frequentemente relacionados a doenças em animais e seres humanos, porém existe crescente preocupação com outros sorotipos como Heidelberg, Senftenberg, Infantis e Minnesota. Estudo recente de Voss-Rech et al. (2011) em amostras provenientes de aviários de frangos de corte do Brasil entre 2009 e 2010 encontrou 20 sorovares, sendo Minnesota o de maior prevalência, representando 37,93\% das amostras. 
A aplicação de práticas de biosseguridade é a maneira mais importante de prevenir Salmonella nos plantéis, associada a outras ferramentas como probióticos, prebióticos, extratos de plantas entre outras. De maneira especial, o mecanismo de ação de probióticos sobre o controle das salmoneloses refere-se à exclusão competitiva (Nurmi \& Rantalla 1973, Borchers et al. 2009) e imunomodulação associada à melhora da integridade na barreira epitelial e na resposta imunológica celular e humoral (Lee et al. 2010).

De fato a colonização bacteriana do trato gastrintestinal tem sido descrita como crucial para o desenvolvimento do sistema imunológico de mucosas e do circuito imuno regulatório sistêmico (Isolauri et al. 2001). Os componentes da parede celular de bactérias como peptidoglicanos e glicopolissacarídeos tem importante papel na ativação inicial do sistema imune. Além disso, a microbiota intestinal esta implicada na manutenção da tolerância imunológica, pois a presença destas bactérias é necessária para manter a hiporesponsividade contra antígenos na mucosa (Gaboriau-Routhiau \& Moreau 1996).

Considerando os potenciais efeitos benéficos dos probióticos sobre o controle de Salmonella sp. e sua interferência na resposta imune das aves, este trabalho teve como objetivo avaliar a resposta imune celular de frangos de corte alimentados com dietas suplementadas com probiótico e desafiados com Salmonella Minnesota (SM).

\section{MATERIAL E MÉTODOS}

Foram utilizados 60 frangos de corte Cobb® de um dia de idade, distribuídos em um delineamento experimental inteiramente casualizado. Ao primeiro dia as aves foram individualmente pesadas e distribuídas em grupos de 20 aves cada, sendo cada ave uma repetição. Os grupos foram alojados em três salas experimentais com isolamento e localizados lado a lado, com pressão negativa e piso coberto com maravalha esterilizada, onde foram mantidas condições ambientais de conforto térmico para as aves durante todo o período experimental. Foi realizado teste de esterilidade nas salas e cama antes do inicio do experimento.

As aves receberam água e ração peletizada ad libitum, sendo a dieta formulada com níveis iguais ou superiores aos recomendados pelo NRC (1994). Os animais foram divididos em três grupos: Controle negativo (CN) - alimentado com dieta basal não inoculado com SM; Controle positivo (CP) - alimentado com dieta basal e inoculados com SM e Probiótico - alimentado com dieta basal adicionada de probiótico (Protexin ${ }^{\mathrm{TM}}$ concentrate) e inoculados com SM. O produto probiótico utilizado era composto de Lactoba-

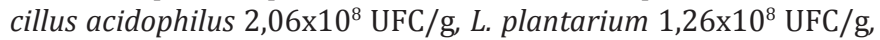

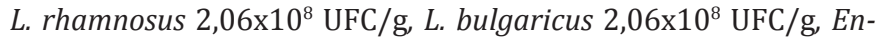
terococcus faecium $6,46 \times 10^{8} \mathrm{UFC} / \mathrm{g}$, Streptococcus thermophilus

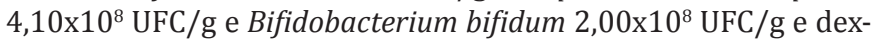
trose $(96,66 \%)$, sendo incorporado à ração na dose $150 \mathrm{~g} / \mathrm{T}$ conforme recomendações do fabricante. Após a peletização a análise de recuperação microbiológica da ração manteve-se dentro dos níveis de garantia do produto.

Na chegada dos animais foi realizada análise de presença/ausência de Salmonella sp. em amostras de fígado e ceco de cinco aves. Aos 14 dias de idade os animais dos grupos Controle positivo e Probiótico foram inoculados por via oral com $1 \mathrm{~mL}$ de solução de SM $\left(10^{8} \mathrm{UFC} / \mathrm{mL}\right)$. Foram coletados suabes de cloaca $48 \mathrm{~h}$ pós-inoculação, amostras de papo e ceco aos sete e 35 dias e amostras de cama aos 21 e 35 dias para contagem de Salmonella sp.
Para a contagem de colônias típicas de Salmonella sp. as amostras foram diluídas em água peptonada $2 \%$ na proporção de 1:9. Uma fração de $1 \mathrm{~mL}$ desta solução foi adicionada em $9 \mathrm{~mL}$ de água peptonada $0,1 \%$ e assim sucessivamente até a diluição $10^{-3}$. Posteriormente, $100 \mu \mathrm{L}$ de cada diluição foram plaqueados em duplicata em meio XLD. As placas foram incubadas a $35^{\circ} \mathrm{C}$ por $24 \mathrm{~h}$ para posterior contagem. A solução inicial de água peptonada $2 \%$ foi incubada a $35^{\circ} \mathrm{C}$ por $24 \mathrm{~h}$ e para as amostras que resultaram em valor nulo na análise quantitativa, $100 \mu \mathrm{L}$ desta solução inicial foram adicionados em $10 \mathrm{~mL}$ de caldo Rappaport-Vassiliadis e incubados a $42^{\circ} \mathrm{C}$ por $24 \mathrm{~h}$ para confirmação de negatividade/ positividade das amostras. Os resultados das contagens de colônias foram expressos conforme os Procedimentos de Contagem de Colônia de acordo com a Normativa no 62 publicada em 26 de agosto de 2003 (Brasil 2003).

Aos 35 dias foram coletados fragmentos de íleo e ceco. A quantificação de células caliciformes foi realizada em fragmentos fixados em formol tamponado 10\%, evidenciadas pela coloração de HE com Alcian Blue. As análises de imunistoquimica para quantificação de células CD4+ e CD8+ foram realizadas em fragmentos congelados em nitrogênio líquido. A quantificação de células caliciformes, CD4+ e CD8+ do epitélio intestinal foi realizada em microscopia óptica (Olympus America Inc., NY, USA), sendo analisados campos com ampliação de 100x (adaptado de Lourenço, 2011).

Os resultados das contagens de colônias de Salmonella sp. foram transformadas em Log 10 para análise estatística paramétrica. Estes dados, bem como os resultados da contagem de células caliciformes, CD4+ e CD8+ foram submetidos à ANOVA seguida de teste de Fischer PLSD a 5\% de probabilidade.

Toda metodologia de estudo foi avaliada e aprovada pelo Comitê de Ética em experimentação animal do Setor de Ciências Agrárias da Universidade Federal do Paraná com Protocolo $n^{\underline{0}}$ 034/2011.

\section{RESULTADOS E DISCUSSÃO}

As amostras de fígado e ceco coletadas no primeiro dia e os papos e cecos coletados aos 7 dias foram negativas para análise de Salmonella sp. Os resultados da análise realizada em suabes de cloaca 48h pós-inoculação (PI) estão expressos no Quadro 1 onde se observa que o grupo Probiótico apresentou redução significativa $(\mathrm{P} \leq 0.05)$ no isolamento de Salmonella sp.

\section{Quadro1. Contagem de colônias de Salmonella sp. $\left(\log _{10}\right)$ em suabes de cloaca de frangos de corte $\mathbf{4 8}$ horas PI nos diferentes grupos}

\begin{tabular}{lc}
\hline \multicolumn{1}{c}{ Grupos } & Salmonella sp. UFC/mL \\
\hline CN & $0,00 \pm 0,00^{\mathrm{b}}$ \\
CP & $3,95 \pm 2,24^{\mathrm{a}}$ \\
Probiótico & $1,45 \pm 1,62^{\mathrm{b}}$ \\
Valor de P & 0,002 \\
\hline
\end{tabular}

Médias ( \pm desvio padrão da média) seguidas de letras distintas na coluna diferem pelo teste de Fischer PLSD a 5\% de probabilidade.

As contagens de Salmonella sp. em papo e ceco aos 35 dias (Quadro 2) demonstram que aves alimentadas com o probiótico apresentaram redução significativa no isolamento de Salmonella sp. no ceco $(46,27 \%)$, mas não no papo, quando comparado ao controle positivo. O ceco é conhecido por abrigar diversos componentes da micro- 
Quadro 2. Contagem de colônias de Salmonella sp.( $\left.\log _{10}\right) \mathrm{em}$ papo e ceco de frangos de corte aos 35 dias nos diferentes grupos

\begin{tabular}{ccc}
\hline Grupos & $\begin{array}{c}\text { Salmonella sp. UFC/g } \\
\text { Papo }\end{array}$ & $\begin{array}{c}\text { Salmonella sp. UFC/g } \\
\text { Ceco }\end{array}$ \\
\hline CN & $0,00 \pm 0,00^{\mathrm{a}}$ & $0,00 \pm 0,00^{\mathrm{a}}$ \\
$\mathrm{CP}$ & $0,87 \pm 0,50^{\mathrm{b}}$ & $4,30 \pm 4,28^{\mathrm{c}}$ \\
Probiótico & $0,73 \pm 0,74^{\mathrm{b}}$ & $2,31 \pm 1,61^{\mathrm{b}}$ \\
Valor de P & 0,025 & 0,001
\end{tabular}

Médias ( \pm desvio padrão da média) seguidas de letras distintas na coluna diferem pelo teste de Fischer PLSD a 5\% de probabilidade.

biota intestinal, sendo também a porção intestinal mais frequentemente colonizada por Salmonella (Fanelli et al. 1971), por possuir condições físico-químicas relativamente constantes e abundante suprimento de nutrientes de fontes endógena e exógena (Revolledo et al. 2006). Higgins et al. (2007) utilizando cepas de bactérias ácido-láticas frente aos sorovares Enteritidis e Typhimurim encontraram diferenças na redução cecal destes microrganismos com variações aproximadas de 65 a 92\% respectivamente, porém os sorovares de Salmonella e a dose de inoculação utilizada foram em menor concentração $\left(10^{4} \mathrm{UFC} / \mathrm{ml}\right)$ do que a utilizada no presente estudo. Em estudo comparativo entre um composto de cepas probióticas e cepas puras de Lactobacillus sp., Higgins et al. (2010) também encontrou diferenças na porcentagem de isolamento de Salmonella Enteritidis $\left(10^{4} \mathrm{UFC} / \mathrm{ml}\right)$, demonstrando que este fator

Quadro 3. Contagem de colônias de Salmonella sp. $\left(\log _{10}\right)$ na cama aos 21 e 35 dias nos diferentes grupos

\begin{tabular}{lcc}
\hline Grupos & $\begin{array}{c}\text { Salmonella sp. UFC/g } \\
\text { 21 dias }\end{array}$ & Salmonella sp. UFC/g \\
\hline CN & $0,00 \pm 0,00^{\mathrm{a}}$ & $0,00 \pm 0,00^{\mathrm{a}}$ \\
CP & $4,30 \pm 0,07^{\mathrm{c}}$ & $3,60 \pm 0,22^{\mathrm{c}}$ \\
Probiótico & $2,90 \pm 1,30^{\mathrm{b}}$ & $2,85 \pm 0,56^{\mathrm{b}}$ \\
Valor de P & 0,001 & 0,001
\end{tabular}

Médias ( \pm desvio padrão da média) seguidas de letras distintas na coluna diferem pelo teste de Fischer PLSD a 5\% de probabilidade. também pode interferir nos resultados encontrados neste estudo. 0 resultado do isolamento de Salmonella na cama das aves (Quadro 3) foi altamente relacionado com os resultados obtidos no ceco, ou seja, aves do grupo que consumiu probiótico na dieta apresentaram redução também na expressão destas bactérias na cama quando comparado ao grupo inoculado que não consumiu probiótico.

Aos 7 dias as aves do grupo Probiótico apresentaram maior expressão de células caliciformes e CD4+ na mucosa de íleo e ceco. No ceco observou-se ainda aumento de células CD8+ (Quadro 4). Vários estudos também demonstram que a presença de bactérias probióticas no trato intestinal das aves pode aumentar a expressão de células CD4+ e CD8+ na mucosa intestinal (Lillehoj \& Chung 1992, Noujain et al. 2008, Mouni et al. 2009, Lee et al. 2010). De fato, o reconhecimento de estruturas da parede celular de bactérias pode induzir o estimulo sobre a imunidade inata que induz o aparecimento de células CD4+ e aparecimento de imunidade específica. $\mathrm{O}$ ambiente cecal é o ambiente ideal para a colonização de muitos microrganismos, como por exemplo, Bifidobacterium bifidum, que tem efeito sobre a expressão de células CD8+ (Gabriel et al., 2006). Antes mesmo disso, a presença de bactérias probióticas pode estimular a proliferação de células produtoras de muco que também são importantes para manter a integridade da mucosa e a barreira contra patógenos na mucosa intestinal (Chichlowski et al. 2007).

Nas análises histológicas realizadas aos 35 dias no íleo e ceco das aves, após desafio microbiológico (Quadro 5), não houve diferença na expressão de células caliciformes e CD4+ entre os tratamentos. Entretanto, aves desafiadas com SM, alimentadas ou não com probióticos, apresentaram número significativamente maior de células CD8+ quando comparadas ao Controle negativo. Segundo Arstila et al. (1994), as células T CD4+ estão envolvidas com a ativação de macrófagos e a modulação da resposta imune, enquanto que células T CD8+ desempenham funções relacionadas com a eliminação do antígeno (Zou et al. 2006). Asheg et al. (2002)

Quadro 4. Contagem de células caliciformes, CD4+ e CD8+ por campo em íleo e ceco de frangos de corte aos 7 dias (aumento de 100x)

\begin{tabular}{lccccccc}
\hline \multirow{2}{*}{ Grupos } & \multicolumn{3}{c}{ Íleo } & & \multicolumn{3}{c}{ Ceco } \\
\cline { 2 - 4 } \cline { 7 - 8 } & Caliciformes & CD4+ & CD8+ & & Caliciformes & CD4+ & CD8+ \\
\hline CN & $41,40 \pm 8,43^{\mathrm{b}}$ & $4,30 \pm 3,71^{\mathrm{b}}$ & $7,90 \pm 2,33$ & & $10,30 \pm 2,34^{\mathrm{b}}$ & $8,30 \pm 3,30^{\mathrm{b}}$ & $10,30 \pm 4,05^{\mathrm{b}}$ \\
Probiótico & $49,35 \pm 6,05^{\mathrm{a}}$ & $9,40 \pm 4,45^{\mathrm{a}}$ & $6,60 \pm 4,70$ & & $14,50 \pm 2,63^{\mathrm{a}}$ & $19,90 \pm 3,54^{\mathrm{a}}$ & $15,20 \pm 3,15^{\mathrm{a}}$ \\
Valor de P & 0,001 & 0,012 & 0,401 & & 0,001 & 0,001 & 0,007
\end{tabular}

Médias ( \pm desvio padrão da média) seguidas de letras distintas na coluna diferem pelo teste de Fischer PLSD a 5\% de probabilidade.

Quadro 5. Contagem de células caliciformes, CD4+ e CD8+ por campo em íleo e ceco de frangos de corte aos 35 dias (aumento de 100x)

\begin{tabular}{lccccccc}
\hline \multirow{2}{*}{ Grupos } & \multicolumn{4}{c}{ Íleo } & & \multicolumn{3}{c}{ Ceco } \\
\cline { 2 - 4 } & Caliciformes & CD4+ & CD8+ & & Caliciformes & CD4+ & CD8+ \\
\hline CN & $64,50 \pm 14,95$ & $16,20 \pm 6,50$ & $6,90 \pm 4,80^{\text {b }}$ & & $10,85 \pm 6,09$ & $19,60 \pm 6,09$ & $11,50 \pm 3,56^{\text {b }}$ \\
CP & $60,95 \pm 12,76$ & $15,50 \pm 5,60$ & $11,40 \pm 6,35^{\text {a }}$ & & $13,70 \pm 5,36$ & $23,70 \pm 8,35$ & $21,90 \pm 5,68^{\text {a }}$ \\
Probiótico & $58,35 \pm 16,69$ & $15,70 \pm 4,30$ & $13,30 \pm 2,83^{a}$ & & $11,90 \pm 4,41$ & $24,60 \pm 4,83$ & $22,40 \pm 6,20^{\text {a }}$ \\
Valor de P & 0,432 & 0,958 & 0,020 & & 0,240 & 0,214 & 0,001
\end{tabular}

Médias ( \pm desvio padrão da média) seguidas de letras distintas na coluna diferem pelo teste de Fischer PLSD a 5\% de probabilidade. 
verificaram aumento de células CD4+ e CD8+ no ceco de aves de 1 dia desafiadas com SE. Estudo de Noujain et al. (2008) verificou aumento de células CD8+ no intestino de aves tratadas com Lactobacillus spp. e microflora cecal, desafiadas com SE, quando comparadas ao Controle negativo. Em nosso estudo o aumento da expressão de células CD8+ neste período pode estar relacionado tanto à presença de probióticos quanto a presença de salmonela, entretanto o menor isolamento de SM de aves alimentadas com probióticos sugere que sua utilização é conveniente para auxiliar no controle de Salmonella em planteis avícolas.

\section{CONCLUSÃO}

A utilização de bactérias probióticas, como Lactobacillus acidophilus, L. plantarium, L. rhamnosus, L. bulgaricus, Enterococcus faecium, Streptococcus thermophilus e Bifidobacterium bifidum, na dieta de frangos de corte aumenta a expressão de células caliciformes, CD4+e CD8+ na mucosa intestinal das aves e reduz o isolamento de Salmonella sp. no ceco das aves após desafio microbiológico.

Agradecimentos.- À empresa Novartis Saúde Animal Ltda pela disponibilização do produto e auxílio durante a pesquisa.

\section{REFERÊNCIAS}

Asheg A., Levkut M., Revajova V., Sevcikova Z., Kolodzieyski L. \& Pistl J. 2002. T lymphocyte subpopulations and B lymphocyte cells in caecum and spleen of chicks infected with Salmonella enteritidis. Acta Histochem. 104:435-439.

Arstila T.P., Vainio 0. \& Lassila 0. 1994. Central role of CD4+ T cells in avian immune response. Poult. Sci. 73:1019-1026.

Borchers A.T., Selmi C., Meyers F.J., Keen C.L. \& Gershwin M.E. 2009. Probiotics and immunity. J. Gastroenterol. 44(1)26-46.

Brasil 2003. Instrução Normativa no 6 publicada em 26 de agosto de 2003. MAPA, Brasília.

Chichlowski M., Croom J., McBride B.W., Havenstein G.B. \& Koci M.D. 2007. Metabolic and prysiological impact of probiotics or direct-fed-microbials on poultry: A brief review of current knowledge. Int. J. Poult. Sci. 6:694-704.

Fanelli M.J., Sadler W.W., Franti C.E. \& Brownell J.R. 1971. Localization of Salmonellae within the intestinal tract of chickens. Avian Dis. 15(2):366375.

Gaboriau-Routhiau V. \& Moreau M.C. 1996. Gut flora allows recovery of oral tolerance to ovalbumin in mice after transient breakdown mediated by cholera toxin or Escherichia coli heat labile enterotoxin. Pediatr. Res. 39:625-629.

Gabriel I., Lessire M., Mallet S. \& Guillot J.F. 2006. Microflora of the digestive tract: Critical factors and consequences for poultry. World Poult. Sci. J. 62:499-511.

Higgins J.P., Higgins S.E., Vicente J.L., Wolfenden A.D., Tellez G. \& Hargis B.M. 2007. Temporal Effects of Lactic Acid Bacteria Probiotic Culture on Salmonella in Neonatal Broilers. Poult. Sci. 86:1662-1666.

Higgins J.P., Higgins S.E., Wolfenden A.D., Henderson S.N., Torres-Rodriguez A., Vicente J.L., Hargis B.M. \& Tellez G. 2010. Effect of lactic acid bacteria probiotic culture treatment timing on Salmonella Enteritidis in neonatal broilers. Poult. Sci. 89:243-247.

Isolauri E., Sutas Y., Kankaanpaa P., Arvilommi H. \& Salminen S. 2001. Probiotics: effects on immunity. Am. J. Clin. Nutr. 73:444S-450S.

Lee K., Lillehoj H.S. \& Siragusa G.R. 2010. Direct-Fed Microbials and Their Impact on the Intestinal Microflora and Immune System of Chickens. J. Poult. Sci. 47:106-104.

Lillehoj H.S. \& Chung K.S. 1992. Postnatal development of T-lymphocyte subpopulations in intestinal intraepithelium and lamina propria in chickens. Vet. Immunol. Immunopathol. 31:347-360.

Lourenço M.C. 2011. Efeito dos mananoligossacarídeos sobre a resposta imunológica de frangos de corte. Dissertação de Mestrado em Ciências Veterinárias, Curso de Pós-Graduação em Ciências Veterinárias, Universidade Federal do Paraná, Curitiba, PR. 55p.

Mouni F., Aissi E., Hernandez J., Gorocica P., Bouquelet S., Zenteno E., Lascurain R. \& Garfias Y. 2009. Effect of Bifidobacterium bifidum DSM 20082 cytoplasmatic fraction on human imune cells. Immunol. Invest. 38(1):104-115.

National Research Concil 1994. Nutrient requirements of poultry. $9^{\text {th }}$ ed. National Academy Press, Washington, D.C.

Noujain J.C., Andreatti Filho L., Lima E.T., Okamoto A.S., Amorim R.L. \& Torres Neto R. 2008. Detection of T lymphocytes in intestine of broiler chicks treated with Lactobacillus spp. and challenged with Salmonella enterica serovar Enteritidis. Poult. Sci. 87:927-933.

Nurmi E. \& Rantala M. 1973. New aspects of Salmonella infection in broiler production. Nature 241:210-211.

Revolledo L., Ferreira A.J.P. \& Mead G.C. 2006. Prospects in Salmonella control: Competitive exclusion, probiotics and enhancement of avian intestinal immunity. J. Appl. Poult. Res. 15:341-351.

Voss-Rech D., Vaz C.S.L., Alves L., Coldebella A., Leão J.A., Rodrigues D. \& Back A. 2011. Caracterização fenotípica e genotípica de Salmonella spp. isoladas de aviários de frangos de corte no Brasil entre 2009 e 2010. Anais Conferência Facta 2011 de Ciência e Tecnologia Avícolas, Santos, SP. (Resumo)

Zou F.C., Jiang Y.P., Nie K., Shang Y.X. \& Li H.L. 2006. Dynamic changes of CD4+ and CD8 T lymphocyte subpopulations in blood of chicks infected with Eimeria tenella. Poult. Husbandry Dis. Control 1:4-7. 